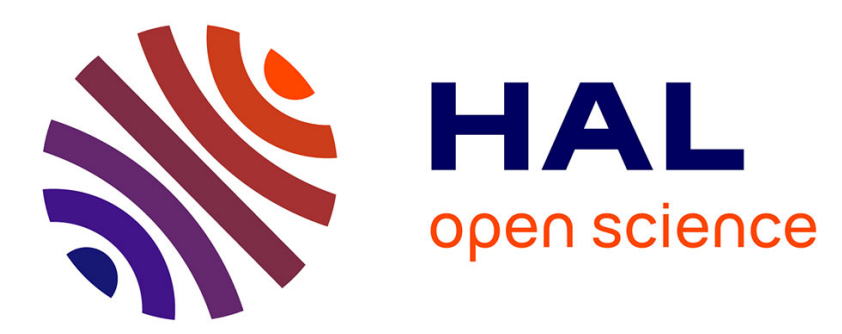

\title{
On a reaction-diffusion model for calcium dynamics in dendritic spines
}

Kamel Hamdache, Mauricio Labadie

\section{To cite this version:}

Kamel Hamdache, Mauricio Labadie. On a reaction-diffusion model for calcium dynamics in dendritic spines. Nonlinear Analysis: Real World Applications, 2009, 10 (4), pp.2478-2492. 10.1016/j.nonrwa.2008.05.005 . hal-00193999v2

\section{HAL Id: hal-00193999 \\ https://hal.science/hal-00193999v2}

Submitted on 15 May 2008

HAL is a multi-disciplinary open access archive for the deposit and dissemination of scientific research documents, whether they are published or not. The documents may come from teaching and research institutions in France or abroad, or from public or private research centers.
L'archive ouverte pluridisciplinaire HAL, est destinée au dépôt et à la diffusion de documents scientifiques de niveau recherche, publiés ou non, émanant des établissements d'enseignement et de recherche français ou étrangers, des laboratoires publics ou privés. 


\title{
On a reaction-diffusion model for calcium dynamics in dendritic spines
}

\author{
Kamel Hamdache ${ }^{1} \quad$ Mauricio Labadie $^{2}$
}

December 5th, 2007

\begin{abstract}
As it was pointed out by D. Holcman and Z. Schuss in Modeling calcium dynamics in dendritic spines (SIAM J. Appl. Math. 2005, Vol. 65, No. 3, pp. 1006-1026), the concentration of calcium ions inside the dendritic spines plays a crucial role in the synaptic plasticity, and in consequence in cognitive processes like learning and memory. The goal of this paper is to study the reactiondiffusion model of calcium dynamics in dendritic spines proposed by Holcman and Schuss. We start from the construction of the model of Holcman and Schuss, taking special care on the hypotheses in order to set a problem with realistic biological assumptions supported by experimental evidence but still mathematically solvable. We show that the dynamics of the calcium ions and the proteins interacting with them follows a system of coupled nonlinear reaction-diffusion equations, which is degenerate elliptic if the proteins are considered fixed, and strongly elliptic if they diffuse with a diffusion coefficient $d>0$. In the first case we prove a priori estimates, global existence, global uniqueness and positivity of solutions, whereas in the second case we prove not only the same features but also that the problem is well-posed. Moreover, we show that there is a "continuous" link between the two problems in the sense that the solutions of the problem with $d>0$ converge to the solutions of the problem with $d=0$.
\end{abstract}

Keywords: Reaction-diffusion equations, calcium dynamics, dendritic spines, global existence, global uniqueness, well-posed problems.

AMS subject classifications: 35K57, 35A05, 46N60, 92Cxx (92C20, 92C40)

\footnotetext{
${ }^{1}$ CMAP, Ecole Polytechnique, CNRS. Route de Saclay 91128 Palaiseau, France. Email: hamdache@cmapx.polytechnique.fr

${ }^{2}$ Correspondig author. CAMS, EHESS, CNRS. 54 Boulevard Raspail 75006 Paris, France. Email: mauricio.labadie@gmail.com, labadie@ehess.fr
} 


\section{Contents}

1 Introduction 3

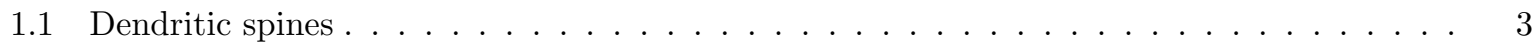

1.2 The role of $\mathrm{Ca}^{2+}$ in spine twitching and synaptic plasticity $\ldots \ldots \ldots \ldots \ldots$

1.3 The original model . . . . . . . . . . . . . . . . . . . . . . 4

2 The modified model and main results $\quad 6$

2.1 New variables . . . . . . . . . . . . . . . . . . . . . . 6

2.2 Modeling the twitching motion of the spine $\ldots \ldots \ldots \ldots \ldots \ldots$

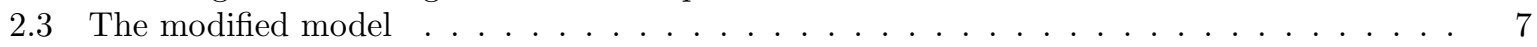

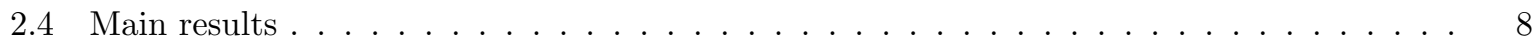

3 Proof of Theorem $1 \quad 10$

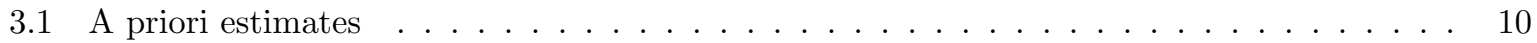

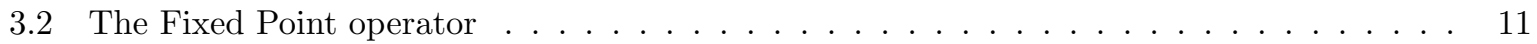

3.3 Conclusion of the proof $\ldots \ldots \ldots \ldots \ldots \ldots$

$4 \begin{array}{ll}\text { Proof of Theorem 2 } & 15\end{array}$

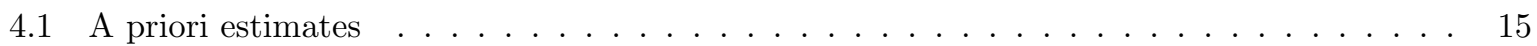

4.2 The Fixed Point operator $\ldots \ldots \ldots \ldots \ldots \ldots \ldots$

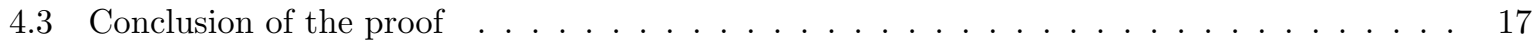

5 Proof of Theorem $3 \quad 19$

6 Final remarks $\quad 20$

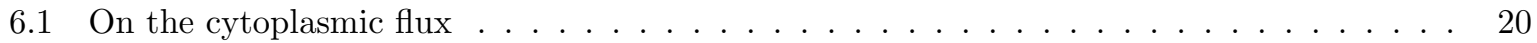

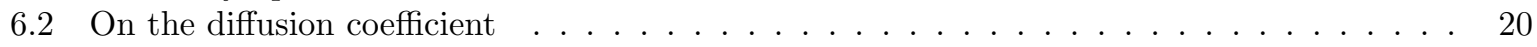

6.3 On the reactions between calcium and the proteins $\ldots \ldots \ldots \ldots \ldots \ldots$

$\begin{array}{lll}7 & \text { Discussion } & 21\end{array}$ 


\section{Introduction}

\section{$1.1 \quad$ Dendritic spines}

Dendritic spines are tiny budlike extensions or protrusions on the dendrites with small bulbous heads and narrow necks. They have lengths around $1 \mu \mathrm{m}$ and volumes around $0.2 \mu \mathrm{m}^{3}$. They are ubiquitous because, on the one hand, $90 \%$ of excitatory synapses occur in dendritic spines, and on the other hand there are more than $10^{13}$ dendritic spines inside the brain. We also find actin microfilaments, endoplasmic reticulum and polyribosomes inside the head, but other structures like mitochondria and microtubules appear to be excluded (see Nimchinsky et al [10]).

Synapses take place on the surface of the spine head where several specialized microstructures can be found, e.g. neuro-receptors and ion channels. Inside the head of the spine there are several molecules related to the intricate biochemical machinery that codifies the information from the pre-synaptic neuron, and eventually, emits a electric potential that flows through the post-synaptic neuron and transmits the excitatory or inhibitory information to another neuron. This means that the former post-synaptic neuron have become pre-synaptic, and the process repeats itself until the signal reaches the target neuron.

As we can see, dendritic spines are at the very basis of the information exchange inside the neural system.

\subsection{The role of $\mathrm{Ca}^{2+}$ in spine twitching and synaptic plasticity}

One of the main questions addressed by Holcman and his colaborators in [6] and [7] was to understand the spine twitching and the synaptic plasticity in terms of the binding reactions between the $\mathrm{Ca}^{2+}$ ions and some proteins inside the spine. They attributed the twitching motion of the spine to the contraction of actin-myosin AM proteins in the following way. They considered that once an AM protein has four $\mathrm{Ca}^{2+}$ ions bound there occurs a local contraction of the AM, and that all local contractions at a given time produce a global contraction of the spine, which has two consequences: first, it generates the rapid twitching motion of the spine, and second, it produces a hydrodynamical movement of the cytoplasmic fluid in the direction of the dendrite. This motion is thus responsible for the transport of ions, not only $\mathrm{Ca}^{2+}$ but also $\mathrm{Na}^{+}$, into the neuron, which constitutes the electric potential we mentioned in Section 1.1 .

Holcman et al [6] also mention that a protein with four $\mathrm{Ca}^{2+}$ ions bound contracts at a fixed rate until one $\mathrm{Ca}^{2+}$ ion unbinds. The contraction due to $\mathrm{Ca}^{2+}$ binding also appears in the works of Farah $e t$ al [3], Klee et al [8] and Shiftman et al [13], but in all three cases the proteins suffer a conformational change each time a $\mathrm{Ca}^{2+}$ ion binds, and not only when they have four $\mathrm{Ca}^{2+}$ ions.

The synaptic plasticity is defined as changes in the synaptic strength, i.e. in the intensity of the signal transmission between two neurons. These changes can be short-term if they occur in the range of milliseconds or minutes, or long-term if their duration is measured in hours, days, weeks or longer. The long-lasting changes in synapses are related to cognitive processes like learning and memory. These changes are divided in two: Long-Term Potentiation (LTP), if there is an increase in the synaptic strength, or Long-Term Depression (LTD), if there is a decrease in the synaptic strength. The major determinant of whether LTP or LTD appears seems to be the amount of $\mathrm{Ca}^{2+}$ in the post-synaptic cell: small rises in $\mathrm{Ca}^{2+}$ lead to depression, whereas large increases trigger potentiation (see Purves et al [12], Chapter 24, pp. 575-610).

As we can see, $\mathrm{Ca}^{2+}$ ions inside the dendritic spine play a crucial role in the twitching motion and synaptic plasticity, and therefore in cognitive processes like learning and memory. 


\subsection{The original model}

The mathematical modeling of calcium dynamics related to neuroscience is a vast topic. Since we will only mention the nonlinear reaction-diffusion model proposed by Holcman and Schuss [7], we recommend [4] for discussions of the different models for calcium dynamics in neurons.

Following Holcman and Schuss [7] we will consider that the $\mathrm{Ca}^{2+}$ ions have a dynamics governed by the Langevin equation

$$
\dot{\boldsymbol{x}}(t)=\boldsymbol{V}(\boldsymbol{x}, t)+\sqrt{2 D} \dot{\boldsymbol{w}}(t) .
$$

where $\boldsymbol{w}(t)$ is a Brownian motion that represents the thermal fluctuations of the medium, $\boldsymbol{V}(\boldsymbol{x}, t)$ is the cytoplasmic flow field and $D$ is the diffusion coefficient

$$
D=\frac{k_{B} T}{m \mu}
$$

with $k_{B}$ the Boltzmann constant, $T$ the temperature, $m$ the mass and $\mu$ the dynamic viscosity. The Langevin equation (1) has a solution $\boldsymbol{x}(t)$ if $\boldsymbol{V}(\boldsymbol{x}, t)$ is Lipschitz and satisfy the growth condition (see Øksendal [11], Theorem 5.2.1, p. 68)

$$
|\boldsymbol{V}(\boldsymbol{x}, t)| \leq C(1+|\boldsymbol{x}|) .
$$

In order to pass from this microscopic description to a macroscopic level we will not be concerned on the dynamics of each $\mathrm{Ca}^{2+}$ ion $\dot{\boldsymbol{x}}(t)$ but rather on the concentration of the $\mathrm{Ca}^{2+}$ ions, which we will denote $M(\boldsymbol{x}, t)$. When normalized, $M(\boldsymbol{x}, t)$ can be seen as the probability density of the $\mathrm{Ca}^{2+}$ ions of the diffusion (1), and in consequence it solves solves the Fokker-Planck equation

$$
\partial_{t} M(\boldsymbol{x}, t)=\nabla \cdot[D \nabla M(\boldsymbol{x}, t)-\boldsymbol{V}(\boldsymbol{x}, t) M(\boldsymbol{x}, t)]
$$

associated to the diffusion (1).

Holcman and Schuss [7] also suppose, as we will do, that there are no obstacles inside the dendritic spine, like organelles and macromolecules. However, it is important to remark that this is only a simplification of the model and not a biological fact because dendritic spines do have organelles, as we mentioned in Section 1.1.

In the model of Holcman and Schuss [7] there is a reaction term that takes into account the binding and unbinding processes (i.e. the association and dissociation processes) between the calcium ions $\mathrm{Ca}^{2+}$ and some fixed proteins inside the spine like calmodulin CaM, actin-myosin AM and calcineurin. These proteins can carry up to four $\mathrm{Ca}^{2+}$ ions. Since we want to keep track of the number of free and bound ions at any time and position, we need to classify the proteins in terms of the number of bound ions.

Define $S^{j}(\boldsymbol{x}, t)$ for $j=0,1,2,3,4$, as the number of proteins containing $j$ bound ions (note that we are not making any distinction between $\mathrm{CaM}, \mathrm{AM}$ and calcineurin). A protein $S^{j}$ can gain or lose one ion at a time with a constant reaction rate $k_{1}$ or $k_{-1}$, respectively. Therefore, the chemical description of $S^{j}$ is

$$
S^{j-1} \underset{k_{-1}}{\stackrel{k_{1}}{\rightleftharpoons}} S^{j} \stackrel{k_{1}}{\rightleftharpoons} S^{j+1}
$$

The Law of Mass Action states that the rate of a reaction is proportional to the product of the concentrations of the reactants. If we take into account that $S^{j}$ has $j$ occupied binding sites and $4-j$ 
free binding sites, and on each one the four reactions given in (3) we use the Law of Mass Action, it follows that the dynamics of $S^{j}$ is given by

$$
\frac{d S^{j}}{d t}=k_{1} M\left[(5-j) S^{j-1}-(4-j) S^{j}\right]-k_{-1}\left[j S^{j}-(j+1) S^{j+1}\right] .
$$

Now let $\Omega$ be the interior of the dendrite, which we will suppose to be a bounded open set in $\mathbb{R}^{2}$ or $\mathbb{R}^{3}$ with a piecewise smooth Lipschitz boundary. Define $\Gamma:=\partial \Omega$ and consider a partition $\Gamma=\Gamma_{a} \cup \Gamma_{r}$, with $\Gamma_{a}$ the "absorbing" part of the boundary and $\Gamma_{r}$ the "reflecting" part. On $\Gamma_{a}$ the ions $M(\boldsymbol{x}, t)$ leave the spine and they never return, which is expressed mathematically as a zero boundary condition. $\Gamma_{a}$ has two components, $\mathrm{Ca}^{2+}$ pumps at the spine head and the bottom of the spine neck (where the ions enter the dendrite). On $\Gamma_{r}$ the ions $M(\boldsymbol{x}, t)$ cannot leave the spine, i.e. if they hit the boundary they rebound, which is modelled as no flux boundary conditions.

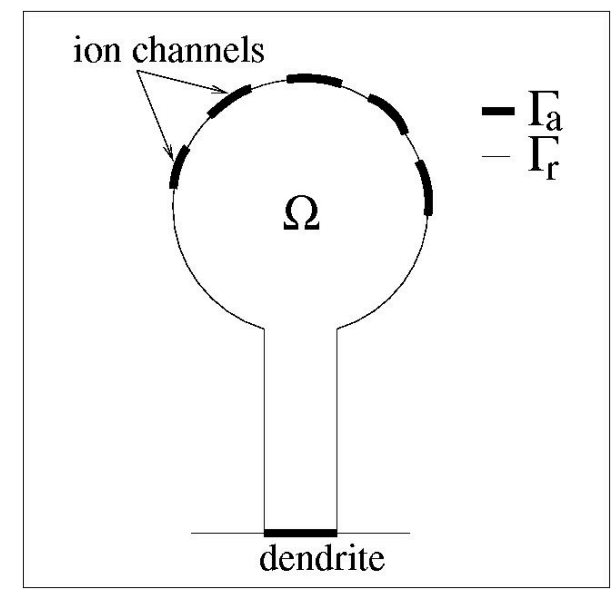

Figure 1: Dendritic spine. We denote $\Omega$ the interior of the spine, $\Gamma$ its surface, $\Gamma_{a}$ the absorbing boundary and $\Gamma_{r}$ the reflecting boundary.

In the light of equations (2) and (3), the reaction-diffusion model of Holcman and Schuss [7] is

$$
\left\{\begin{aligned}
\partial_{t} M & =\nabla \cdot[D \nabla M-\boldsymbol{V} M]-k_{1} M\left[\sum_{j=0}^{4}(4-j) S^{j}\right]+k_{-1}\left[\sum_{j=0}^{4} j S^{j}\right] \\
\partial_{t} S^{j} & =k_{1} M\left[(5-j) S^{j-1}-(4-j) S^{j}\right]-k_{-1}\left[j S^{j}(\boldsymbol{x}, t)-(j+1) S^{j+1}\right] \\
\mathbf{V} & =\nabla \phi, \quad \triangle \phi=0
\end{aligned}\right.
$$


Here, by convention, $S^{-1}(\boldsymbol{x}, t)=S^{5}(\boldsymbol{x}, t)=0$. The initial conditions for the system $(5)$ are

$$
M(\boldsymbol{x}, 0)=m_{0}(\boldsymbol{x}) \geq 0 ; \quad S^{0}(\boldsymbol{x}, 0)=\frac{1}{4} A(\boldsymbol{x}) \geq 0, \quad S^{j}(\boldsymbol{x}, 0)=0 \quad \text { for } j=1,2,3,4,
$$

whereas the boundary conditions are

$$
\left\{\begin{aligned}
M(\sigma, t) & =0 & & \text { on } \Gamma_{a} \times[0, T], \\
(D \nabla M-\boldsymbol{V} M) \cdot \boldsymbol{n}(\sigma, t) & =0 & & \text { on } \Gamma_{r} \times[0, T], \\
\nabla \phi \cdot \boldsymbol{n}(\sigma, t) & =a(\sigma) \lambda(t) & & \text { on } \Gamma \times[0, T],
\end{aligned}\right.
$$

where $\boldsymbol{n}(\sigma, t)$ is the outer normal of $\Gamma, a(\sigma)$ is given, and $\lambda(t)$ is related to the total number of $\mathrm{Ca}^{2+}$ ions bound to the proteins at time $t$.

\section{The modified model and main results}

\section{$2.1 \quad$ New variables}

From the system (5) we observe that the important variables for the description of the dynamics for $M(\boldsymbol{x}, t)$ are not the proteins $S^{j}(\boldsymbol{x}, t)$ but the quantities

$$
U(\boldsymbol{x}, t)=\sum_{j=0}^{4}(4-j) S^{j}(\boldsymbol{x}, t), \quad W(\boldsymbol{x}, t)=\sum_{j=0}^{4} j S^{j}(\boldsymbol{x}, t) .
$$

Note that $U(\boldsymbol{x}, t)$ is the total number of free binding sites about $\boldsymbol{x}$ at time $t$, and $W(\boldsymbol{x}, t)$ is the total number of occupied binding sites. This change of variables not only simplifies the notation but also reduces the system (5) to a new system on the variables $(M, U, W)$. Indeed, if we develop the equations $S^{j}(\boldsymbol{x}, t)$ in the system (5) it follows that

$$
\begin{aligned}
\partial_{t} S^{0} & =k_{1} M\left[0-4 S^{0}\right]-k_{-1}\left[0-1 S^{1}\right], \\
\partial_{t} S^{1} & =k_{1} M\left[4 S^{0}-3 S^{1}\right]-k_{-1}\left[1 S^{1}-2 S^{2}\right], \\
\partial_{t} S^{2} & =k_{1} M\left[3 S^{1}-2 S^{2}\right]-k_{-1}\left[2 S^{2}-3 S^{3}\right], \\
\partial_{t} S^{3} & =k_{1} M\left[2 S^{2}-1 S^{1}\right]-k_{-1}\left[3 S^{3}-4 S^{4}\right], \\
\partial_{t} S^{4} & =k_{1} M\left[1 S^{1}-0\right]-k_{-1}\left[4 S^{4}-0\right] .
\end{aligned}
$$

Multiplying the $j$-th equation by $(4-j)$ and adding them up we obtain

$$
\partial_{t} U=-k_{1} M U+k_{-1} W .
$$

Analogously, multiplying by $j$ and adding up we get

$$
\partial_{t} W=k_{1} M U-k_{-1} W .
$$

In the variables $U$ and $W$ the equation for $M(\boldsymbol{x}, t)$ takes the form

$$
\partial_{t} M=\nabla \cdot[D \nabla M-\boldsymbol{V} M]-k_{1} M U+k_{-1} W .
$$




\subsection{Modeling the twitching motion of the spine}

In Section 1.2 we mentioned that each time a $\mathrm{Ca}^{2+}$ ion binds to a protein this latter suffers a contraction, and that the addition of all these local contractions have two effects: the twitching of the spine and changes in the cytoplasmic flow field $\boldsymbol{V}(\boldsymbol{x}, t)$. In order to take into account both effects we will assume that the spine movement depends on the cytoplasmic velocity at the spine surface $\Gamma$, and that this value depends on the total number of $\mathrm{Ca}^{2+}$ ions that are bound to the proteins. More precisely, if we define

$$
\lambda(t):=\int_{\Omega} W(\boldsymbol{x}, t) d \Omega,
$$

which is the total number of occupied binding sites at time $t$, then our assumption is that the spine surface $\Gamma$ moves with velocity $\boldsymbol{V} \cdot \boldsymbol{n}$ proportional to $\lambda(t)$.

It is worth to mention that Holcman and Schuss [7] supposed that the contraction of a protein takes place only if it has four $\mathrm{Ca}^{2+}$ ions bound, which implies that

$$
\lambda(t)=\int_{\Omega} S^{4}(\boldsymbol{x}, t) d \Omega .
$$

However, as we have already mentioned Section 1.2, assuming (12) instead of (13) is biologically more accurate.

Following Holcman and Schuss [7] we will also suppose that there exists a potential $\phi(\boldsymbol{x}, t)$ such that $\boldsymbol{V}=\nabla \phi$, whose dynamics is given by the equation

$$
\left\{\begin{aligned}
\Delta \phi(\boldsymbol{x}, t) & =0 & & \text { on } \Omega \times[0, T] \\
\nabla \phi \cdot \boldsymbol{n}(\sigma, t) & =a(\sigma) \lambda(t) & & \text { on } \Gamma \times[0, T]
\end{aligned}\right.
$$

where $a(\sigma) \in L^{\infty}(\Gamma)$ is given, together with the orthogonality condition

$$
\int_{\Omega} \phi(\boldsymbol{x}, t) d \Omega=0
$$

and the compatibility condition

$$
\int_{\Gamma} a(\sigma, t) d S=0 .
$$

For a fixed $t \geq 0$ the problem (14) is the Laplace equation with Neumann boundary conditions; therefore the solution $\phi$ exists, and is unique due to the orthogonality condition (15). Moreover, using integration by parts and Poincaré's inequality it can be shown that there is a constant $C>0$ such that

$$
\|\boldsymbol{V}(t)\|_{\left[L^{2}(\Omega)\right]^{n}} \leq C\|W\| \quad \text { for all } t \in[0, T],
$$

where $\|\cdot\|$ denotes the norm in $L^{2}(\Omega)$.

\subsection{The modified model}

Observe that (9) and (10) imply that for all times

$$
U(\boldsymbol{x}, t)+W(\boldsymbol{x}, t)=4 \sum_{j=0}^{4} S^{j}(\boldsymbol{x}, 0)=A(\boldsymbol{x}),
$$


so we can reduce further the system (9)-(11) to

$$
\left\{\begin{aligned}
\partial_{t} M & =\nabla \cdot[D \nabla M-\boldsymbol{V} M]-k_{1} M U+k_{-1}[A-U] \\
\partial_{t} U & =-k_{1} M U+k_{-1}[A-U] \\
\mathbf{V} & =\nabla \phi, \quad \triangle \phi=0 .
\end{aligned}\right.
$$

with initial conditions

and boundary conditions

$$
\left\{\begin{aligned}
M(\boldsymbol{x}, 0) & =m_{0}(\boldsymbol{x}) \\
U(\boldsymbol{x}, 0) & =A(\boldsymbol{x})
\end{aligned}\right.
$$

$$
\left\{\begin{aligned}
M(\sigma, t) & =0 & & \text { on } \Gamma_{a} \times[0, T], \\
(D \nabla M-\boldsymbol{V} M) \cdot \boldsymbol{n}(\sigma, t) & =0 & & \text { on } \Gamma_{r} \times[0, T], \\
\nabla \phi \cdot \boldsymbol{n}(\sigma, t) & =a(\sigma) \lambda(t) & & \text { on } \Gamma \times[0, T] .
\end{aligned}\right.
$$

There is an important issue we want to remark. So far we have considered that the proteins were fixed in the cytoplasm, but this is not true in the real biological situation. In order to take into account the motion of the proteins we can suppose that they diffuse with a constant diffusion coefficient $d>0$ and that they cannot leave the spine. Under these assumptions, the model (18)-(20) with diffusive proteins takes the form

$$
\left\{\begin{aligned}
\partial_{t} M & =\nabla \cdot[D \nabla M-\boldsymbol{V} M]-k_{1} M U+k_{-1} W \\
\partial_{t} U & =d \triangle U-k_{1} M U+k_{-1} W \\
\partial_{t} W & =d \triangle U+k_{1} M U-k_{-1} W \\
\mathbf{V} & =\nabla \phi, \quad \triangle \phi=0
\end{aligned}\right.
$$

with initial conditions

$$
\left\{\begin{aligned}
M(\boldsymbol{x}, 0) & =m_{0}(\boldsymbol{x}) \\
U(\boldsymbol{x}, 0) & =A(\boldsymbol{x}) \\
W(\boldsymbol{x}, 0) & =0
\end{aligned}\right.
$$

and boundary conditions

$$
\left\{\begin{aligned}
M(\sigma, t) & =0 & & \text { on } \Gamma_{a} \times[0, T], \\
(D \nabla M-\boldsymbol{V} M) \cdot \boldsymbol{n}(\sigma, t) & =0 & & \text { on } \Gamma_{r} \times[0, T], \\
\nabla U \cdot \boldsymbol{n}(\sigma, t) & =0 & & \text { on } \Gamma \times[0, T], \\
\nabla W \cdot \boldsymbol{n}(\sigma, t) & =0 & & \text { on } \Gamma \times[0, T], \\
\nabla \phi \cdot \boldsymbol{n}(\sigma, t) & =a(\sigma) \lambda(t) & & \text { on } \Gamma \times[0, T] .
\end{aligned}\right.
$$

Note that $d$ should be much smaller than $D$ because the proteins we are considering are around $10^{6}$ times bigger than the calcium ions.

\subsection{Main results}

From now on we will always assume the following hypotheses:

$$
\left\{\begin{array}{l}
m_{0}(\boldsymbol{x}) \in L^{\infty}(\Omega), \quad m_{0}(\boldsymbol{x}) \geq 0 \quad \text { a.e. in } \Omega \\
A(\boldsymbol{x}) \in L^{\infty}(\Omega), \quad A(\boldsymbol{x}) \geq 0 \quad \text { a.e. in } \Omega \\
a(\sigma) \in L^{\infty}(\Gamma), \quad \int_{\Gamma} a(\sigma) d S=0
\end{array}\right.
$$

For the model with fixed proteins (18)-(20) we prove global existence, global uniqueness, boundedness and positivity of solutions. 
Theorem 1 For any $T>0$ the reaction-diffusion system (18)-(20) has global unique weak solutions $M(\boldsymbol{x}, t), U(\boldsymbol{x}, t)$ and $\boldsymbol{V}(\boldsymbol{x}, t)$ on $\Omega \times(0, T)$ with the following properties:

1. $M \in L^{\infty}(\Omega \times(0, T))$ and $0 \leq M(\boldsymbol{x}, t) \leq\left\|m_{0}\right\|_{\infty}+k_{-1} t\|A\|_{\infty}$ a.e. in $\Omega \times(0, T)$.

2. $M \in L^{\infty}\left(0, T ; H^{1}(\Omega)\right)$ and

$$
\|M(t)\|^{2}+D \int_{0}^{t} e^{C(t-s)}\|\nabla M(s)\|^{2} d s \leq e^{C t}\left[\left\|m_{0}\right\|^{2}+k_{-1}^{2} t\|A\|^{2}\right] .
$$

3. $U \in L^{\infty}(\Omega \times(0, T))$ and $0 \leq U(\boldsymbol{x}, t) \leq A(\boldsymbol{x})$ a.e. in $\Omega \times(0, T)$.

4. $\boldsymbol{V} \in L^{\infty}\left(0, T ;\left[L^{2}(\Omega)\right]^{n}\right)$ and $\|\boldsymbol{V}\|_{L^{\infty}\left(0, T ;\left[L^{2}(\Omega)\right]^{n}\right)} \leq C\|a\|_{\infty}\|A\|_{\infty}$.

For the model with diffusive proteins (18)-(20) the situation is even nicer because we have not only the same results of Theorem 1 but in addition the problem is well-posed.

Theorem 2 For any $T>0$ the reaction-diffusion system (21)-(23) is well-posed, i.e. it has global unique weak solutions $M(\boldsymbol{x}, t), U(\boldsymbol{x}, t), W(\boldsymbol{x}, t)$ and $\boldsymbol{V}(\boldsymbol{x}, t)$ on $\Omega \times(0, T)$ depending continuously on the initial data. Moreover, we have the following properties:

1. $M \in L^{\infty}(\Omega \times(0, T))$ and $0 \leq M(\boldsymbol{x}, t) \leq\left\|m_{0}\right\|_{\infty}+k_{-1} t\|A\|_{\infty}$ a.e. in $\Omega \times(0, T)$.

2. $M \in L^{\infty}\left(0, T ; H^{1}(\Omega)\right)$ and

$$
\|M(t)\|^{2}+D \int_{0}^{t} e^{C(t-s)}\|\nabla M(s)\|^{2} d s \leq e^{C t}\left[\left\|m_{0}\right\|^{2}+k_{-1}^{2} t\|A\|^{2}\right] .
$$

3. $U, W \in L^{\infty}(\Omega \times(0, T))$, they are non-negative and $0 \leq U(\boldsymbol{x}, t)+W(\boldsymbol{x}, t) \leq A(\boldsymbol{x})$ a.e. in $\Omega \times(0, T)$.

4. $U, W \in L^{\infty}\left(0, T ; H^{1}(\Omega)\right)$ and

$$
\|U(t)\|^{2}+\|W(t)\|^{2}+2 d \int_{0}^{t} e^{\int_{s}^{t} c(r) d r}\left(\|\nabla U(s)\|^{2}+\|\nabla W(s)\|^{2}\right) d s \leq e^{\int_{0}^{t} c(s) d s}\|A\|^{2}
$$

where $c(t)=2\left[k_{-1}+k_{1} \alpha(t)\right]$ and $\alpha(t)=\left\|m_{0}\right\|_{\infty}+k_{-1} t\|A\|_{\infty}$.

5. $\boldsymbol{V} \in L^{\infty}\left(0, T ;\left[L^{2}(\Omega)\right]^{n}\right)$ and $\|\boldsymbol{V}\|_{L^{\infty}\left(0, T ;\left[L^{2}(\Omega)\right]^{n}\right)} \leq C\|a\|_{\infty}\|A\|_{\infty}$

There is a link between the model with fixed proteins (18)-(20) and the model with diffusive proteins (21)-(23). Indeed, we have the following "continuity" result.

Theorem 3 If $d \rightarrow 0$ then the sequence $\left(M^{d}, U^{d}, W^{d}, \mathbf{V}^{d}\right)$ of solutions of (21)-(23) converges to the solution $(M, U, W, \mathbf{V})$ of (18)-(20) in the following senses:

1. $M^{d}, U^{d}$ and $W^{d}$ converge weakly in $L^{2}\left(0, T ; L^{2}(\Omega)\right)$ to $M, U$ and $W$, respectively.

2. $\mathbf{V}^{d}$ converges to $\mathbf{V}$ weakly in $L^{2}\left(0, T ;\left[L^{2}(\Omega)\right]^{n}\right)$.

3. $M^{d}$ converges strongly in $L^{2}\left(0, T ; L^{2}(\Omega)\right)$ to $M$.

4. $U^{d}$ and $W^{d}$ converge weakly-^ in $L^{\infty}(\Omega \times(0, T))$ to $U$ and $W$, respectively.

5. In the limit we have $U(\boldsymbol{x}, t)+W(\boldsymbol{x}, t)=A(\boldsymbol{x})$ a.s. in $\Omega \times(0, T)$. 


\section{Proof of Theorem 1}

\subsection{A priori estimates}

Lemma 1 If $M(\boldsymbol{x}, t)$ is a solution of (18)-(20) such that $M(\boldsymbol{x}, t) \geq 0$ a.e. in $\Omega \times(0, T)$ then:

1. $0 \leq U(\boldsymbol{x}, t) \leq A(\boldsymbol{x})$ a.e. In particular, $U(\boldsymbol{x}, t) \in L^{\infty}(\Omega \times(0, T))$.

2. $M(\boldsymbol{x}, t) \in L^{\infty}(\Omega \times(0, T))$ and $M(\boldsymbol{x}, t) \leq \alpha(t):=\left\|m_{0}\right\|_{\infty}+k_{-1} t\|A\|_{\infty}$ a.e.

3. There exists a positive constant $C=C\left(D,\|\boldsymbol{V} \cdot \boldsymbol{n}\|_{\infty}, \Omega\right)$ such that

$$
\|M(t)\|^{2}+D \int_{0}^{t} e^{C(t-s)}\|\nabla M(s)\|^{2} d s \leq e^{C t}\left[\left\|m_{0}\right\|^{2}+k_{-1}^{2} t\|A\|^{2}\right] .
$$

\section{Proof:}

1. Using the equations (18)-(20) we have

$$
\begin{aligned}
U(\boldsymbol{x}, t) & =A(\boldsymbol{x}) \exp \left\{-\int_{0}^{t}\left[k_{1} M(\boldsymbol{x}, s)+k_{-1}\right] d s\right\} \\
& +k_{-1} A(\boldsymbol{x}) \int_{0}^{t} \exp \left\{-\int_{s}^{t}\left[k_{1} M(\boldsymbol{x}, r)+k_{-1}\right] d r\right\} d s .
\end{aligned}
$$

Therefore $U(\boldsymbol{x}, t) \geq 0$ a.e., and using $M(\boldsymbol{x}, t) \geq 0$ a.e. it follows that $U(\boldsymbol{x}, t) \leq A(\boldsymbol{x})$ a.e.

2. Let $\alpha(t)$ be a smooth function and define $Z(\boldsymbol{x}, t):=M(\boldsymbol{x}, t)-\alpha(t)$. Then the equation for $Z(\boldsymbol{x}, t)$ is

$$
\begin{aligned}
\partial_{t} Z-\nabla \cdot(D \nabla Z-\boldsymbol{V} Z)+k_{1} Z U & =-\alpha^{\prime}(t)-k_{1} \alpha(t) U+k_{-1}(A-U), \\
Z(\boldsymbol{x}, 0) & =m_{0}(\boldsymbol{x})-\alpha(0), \\
Z(\sigma, t) & =-\alpha(t) \quad \text { on } \Gamma_{a} \times[0, T], \\
(D \nabla Z-\boldsymbol{V Z}) \cdot \boldsymbol{n}(\sigma, t) & =0 \quad \text { on } \Gamma_{r} \times[0, T],
\end{aligned}
$$

Choosing $\alpha(t):=\left\|m_{0}\right\|_{\infty}+k_{-1} t\|A\|_{\infty}$ it follows that

$$
\begin{aligned}
\partial_{t} Z-\nabla \cdot(D \nabla Z-\boldsymbol{V} Z)+k_{1} Z U & \leq 0, & & \\
Z(\boldsymbol{x}, 0) & \leq 0, & & \\
Z(\sigma, t) & \leq 0 & & \text { on } \Gamma_{a} \times[0, T], \\
(D \nabla Z-\boldsymbol{V} Z) \cdot \boldsymbol{n}(\sigma, t) & =0 & & \text { on } \Gamma_{r} \times[0, T] .
\end{aligned}
$$

Therefore, the Maximum Principle implies that $Z(\boldsymbol{x}, t) \leq 0$ a.e.

3. Multiply (18)-(20) by $M$, integrate over $\Omega$ and use integration by parts to get

$$
\begin{aligned}
\frac{1}{2} \frac{d}{d t} M^{2}+D\|\nabla M\|^{2} & =\frac{1}{2} \int_{\Gamma} \boldsymbol{V} \cdot \boldsymbol{n} M^{2} d S-k_{1} \int_{\Omega} U M^{2} d \Omega \\
& +k_{-1} \int_{\Omega}[A-U] M d \Omega .
\end{aligned}
$$


For the first integral, notice that $\boldsymbol{V} \cdot \boldsymbol{n} \in L^{\infty}(\Gamma \times[0, T])$. Therefore, using trace estimates we obtain that for any $\varepsilon>0$ there exists a constant $C_{1}=C_{1}\left(\varepsilon,\|\boldsymbol{V} \cdot \boldsymbol{n}\|_{\infty}, \Omega\right)$ such that

$$
\frac{1}{2}\left|\int_{\Gamma} \boldsymbol{V} \cdot \boldsymbol{n} M^{2} d S\right| \leq \varepsilon \int_{\Omega}|\nabla M|^{2} d \Omega+\frac{C_{1}}{2} \int_{\Omega}|M|^{2} d \Omega .
$$

For the second and third integrals, observe that $U \geq 0$ since $M \geq 0$, and in consequence

$$
k_{-1} \int_{\Omega}[A-U] M d \Omega \leq \frac{k_{-1}^{2}}{2} \int_{\Omega} A^{2} d \Omega+\frac{1}{2} \int_{\Omega} M^{2} d \Omega .
$$

Choosing $C:=C_{1}+1$ and $\varepsilon=D / 2$ we obtain

$$
\frac{d}{d t}\|M\|^{2}+D\|\nabla M\|^{2} \leq C\|M\|^{2}+k_{-1}^{2}\|A\|^{2} .
$$

Finally, multiplying by $e^{-C t}$ and integrating on $[0, t]$ we obtain the result.

\subsection{The Fixed Point operator}

Define

$$
\mathcal{K}:=\left\{M \in L^{2}\left(0, T ; L^{2}(\Omega)\right): M(\boldsymbol{x}, t) \geq 0 \quad \text { a.e. }\right\}
$$

Fix $M^{\sharp} \in \mathcal{K}$ and set

$$
\partial_{t} U=-k_{1} M^{\sharp} U+k_{-1}[A-U], \quad U(\boldsymbol{x}, 0)=A(\boldsymbol{x}) .
$$

For any finite time interval $[0, T]$ this linear problem has a unique solution $U(\boldsymbol{x}, t)$ given by

$$
\begin{aligned}
U(\boldsymbol{x}, t) & =A(\boldsymbol{x}) \exp \left\{-\int_{0}^{t}\left[k_{1} M^{\sharp}(\boldsymbol{x}, s)+k_{-1}\right] d s\right\} \\
& +k_{-1} A(\boldsymbol{x}) \int_{0}^{t} \exp \left\{-\int_{s}^{t}\left[k_{1} M^{\sharp}(\boldsymbol{x}, r)+k_{-1}\right] d r\right\} d s,
\end{aligned}
$$

which satisfies $0 \leq U(\boldsymbol{x}, t) \leq A(\boldsymbol{x})$ a.e. in $\Omega \times(0, T)$. With this $U(\boldsymbol{x}, t)$ define

$$
\lambda(t):=\int_{\Omega}[A(\boldsymbol{x})-U(\boldsymbol{x}, t)] d \Omega
$$

and set the elliptic problem

$$
\left\{\begin{aligned}
\triangle \phi(\boldsymbol{x}, t) & =0 & & \text { in } \Omega, \\
\nabla \phi \cdot \boldsymbol{n}(\sigma, t) & =a(\sigma) \lambda(t) & & \text { on } \Gamma,
\end{aligned}\right.
$$

with $a(\sigma) \in L^{\infty}(\Gamma)$ and $\int_{\Omega} \phi(\boldsymbol{x}, t) d \Omega=\int_{\Gamma} a(\sigma) d S=0$. This linear problem has a unique solution $\phi(\boldsymbol{x}, t) \in L^{\infty}\left(0, T ; H^{1}(\Omega)\right)$ satisfying

$$
\|\phi\|_{H^{1}(\Omega)} \leq C\|a\|_{\infty}\|A\|_{\infty} .
$$

Now, define $\boldsymbol{V}(\boldsymbol{x}, t)=\nabla \phi(\boldsymbol{x}, t)$ and set the linear problem

$$
\begin{aligned}
\partial_{t} M=\nabla \cdot[D \nabla M-\boldsymbol{V} M]-k_{1} M U+k_{-1}[A-U] & \text { in } \Omega \times(0, T), \\
M(\sigma, t)=0 & \text { on } \Gamma_{a} \times[0, T], \\
\nabla M \cdot \boldsymbol{n}(\sigma, t)=0 & \text { on } \Gamma_{r} \times[0, T], \\
M(\boldsymbol{x}, 0)=m_{0}(\boldsymbol{x}) & \text { in } \Omega .
\end{aligned}
$$


For any finite time interval $[0, T]$ there exists a unique weak solution $M(\boldsymbol{x}, t)$ satisfying the estimates in Lemma 1.

In summary, we have just constructed a chain of maps $M^{\sharp} \mapsto U \mapsto \boldsymbol{V} \mapsto M$, where each map is given by a solution of a differential equation. In the light of this, we can define the operator $\mathcal{R}\left(M^{\sharp}\right):=M$, and our task now is to show that $\mathcal{R}$ has a fixed point.

In order to apply Schauder's Fixed Point Theorem to the operator $\mathcal{R}$ we need to show that it satisfies the following conditions.

Lemma 2 Fix a positive time $T>0$. Then:

1. $\mathcal{K}$ is a convex closed subset of $L^{2}\left(0, T ; L^{2}(\Omega)\right)$.

2. $\mathcal{R}: \mathcal{K} \rightarrow \mathcal{K}$.

3. $\mathcal{R}: L^{2}\left(0, T ; L^{2}(\Omega)\right) \rightarrow L^{2}\left(0, T ; L^{2}(\Omega)\right)$ is continuous.

4. $\mathcal{R}(\mathcal{K})$ is relatively compact in $L^{2}\left(0, T ; L^{2}(\Omega)\right)$.

\section{Proof:}

1. $\mathcal{K}$ is a convex closed subset of $L^{2}\left(0, T ; L^{2}(\Omega)\right)$. It is immediate.

2. $\mathcal{R}: \mathcal{K} \rightarrow \mathcal{K}$. Multiply (18)-(20) by $-M^{-}$, integrate over $\Omega$ and use integration by parts. After those calculation we arrive to

$$
\begin{aligned}
\frac{1}{2} \frac{d}{d t}\left\|M^{-}\right\|^{2}+D\left\|\nabla M^{-}\right\|^{2}= & \frac{1}{2} \int_{\Gamma} \boldsymbol{V} \cdot \boldsymbol{n}\left|M^{-}\right|^{2} d S-k_{1} \int_{\Omega} U\left|M^{-}\right|^{2} d \Omega \\
& -k_{-1} \int_{\Omega}[A-U] M^{-} d \Omega .
\end{aligned}
$$

From Lemma 1 we have $0 \leq U \leq A$ since $M^{\sharp} \in \mathcal{K}$. Using this fact and trace estimates we arrive to

$$
\frac{1}{2} \frac{d}{d t}\left\|M^{-}\right\|^{2}+(D-\varepsilon)\left\|\nabla M^{-}\right\|^{2} \leq C\left\|M^{-}\right\|^{2},
$$

where $\varepsilon>0$ is arbitrary. Therefore $M^{-}(\boldsymbol{x}, t) \equiv 0$ a.e. in $\Omega \times(0, T)$, and in consequence $M \in \mathcal{K}$.

3. $\mathcal{R}: L^{2}\left(0, T ; L^{2}(\Omega)\right) \rightarrow L^{2}\left(0, T ; L^{2}(\Omega)\right)$ is continuous. Let $M_{1}^{\sharp}, M_{2}^{\sharp} \in \mathcal{K}$, and for each $i=1,2$ consider the chain of maps

$$
M_{i}^{\sharp} \longmapsto U_{i} \longmapsto V_{i} \longmapsto M_{i} .
$$

Define $\hat{M}^{\sharp}:=M_{2}^{\sharp}-M_{1}^{\sharp}, \hat{U}:=U_{2}-U_{1}, \hat{\varphi}:=\varphi_{2}-\varphi_{1}, \hat{\boldsymbol{V}}:=\boldsymbol{V}_{2}-\boldsymbol{V}_{1}$ and $\hat{M}:=M_{2}-M_{1}$. The differences $\hat{M}$ and $\hat{U}$ solve the equations

$$
\begin{aligned}
\partial_{t} \hat{M} & =\nabla \cdot\left[D \nabla \hat{M}-\boldsymbol{V}_{1} \hat{M}-\hat{\boldsymbol{V}} M_{2}\right]-\left[k_{1} M_{2}+k_{-1}\right] \hat{U}+k_{1} \hat{M} U_{1}, \\
\partial_{t} \hat{U} & =-\left[k_{1} M_{2}^{\sharp}+k_{-1}\right] \hat{U}+k_{1} \hat{M^{\sharp}} U_{1},
\end{aligned}
$$

with homogeneous boundary and initial conditions, whereas $\hat{\boldsymbol{V}}$ solves

$$
\begin{aligned}
\nabla \cdot \hat{\boldsymbol{V}} & =0 \\
\hat{\boldsymbol{V}} \cdot \boldsymbol{n} & =-a \int_{\Omega} \hat{U} d \Omega .
\end{aligned}
$$


We can solve explicitly the equation (27):

$$
\hat{U}(\boldsymbol{x}, t)=k_{1} \int_{0}^{t} \exp \left\{-\int_{s}^{t}\left[k_{1} M_{2}^{\sharp}(\boldsymbol{x}, r)+k_{-1}\right] d r\right\} \hat{M}^{\sharp}(\boldsymbol{x}, s) U_{1}(\boldsymbol{x}, s) d s .
$$

Since $M_{2}^{\sharp} \geq 0$ and $0 \leq U_{1} \leq A$ it follows that

$$
|\hat{U}(\boldsymbol{x}, t)| \leq k_{1} A(\boldsymbol{x}) \int_{0}^{t}\left|\hat{M}^{\sharp}(\boldsymbol{x}, s)\right| d s .
$$

Using Hölder's inequality we find that

$$
|\hat{U}(\boldsymbol{x}, t)|^{2} \leq k_{1}^{2}\|A\|_{\infty}^{2} t \int_{0}^{t}\left|\hat{M}^{\sharp}(\boldsymbol{x}, s)\right|^{2} d s,
$$

and integrating over $\Omega$ we obtain

$$
\|\hat{U}(t)\|^{2} \leq \beta(t)\left\|\hat{M}^{\sharp}\right\|_{L^{2}\left(0, T ; L^{2}(\Omega)\right)}^{2} ; \quad \beta(t)=k_{1}^{2}\|A\|_{\infty}^{2} t .
$$

Multiply the equation (26) by $\hat{M}$, integrate over $\Omega$ and use integration by parts to obtain

$$
\begin{aligned}
\frac{1}{2} \frac{d}{d t}\|\hat{M}\|^{2}+D\|\nabla \hat{M}\|^{2}= & \frac{1}{2} \int_{\Gamma} \boldsymbol{V}_{1} \cdot \boldsymbol{n}|\hat{M}|^{2} d S-\int_{\Omega} \hat{\boldsymbol{V}} \cdot \nabla \hat{M} M_{2} d \Omega \\
& -\int_{\Omega}\left[k_{1} M_{2}+k_{-1}\right] \hat{U} \hat{M} d \Omega-k_{1} \int_{\Omega} U_{1}|\hat{M}|^{2} d \Omega .
\end{aligned}
$$

Let us estimate the right-hand side of (30). For the first integral, using $\boldsymbol{V}_{1} \cdot \boldsymbol{n} \in L^{\infty}((0, T) \times \Omega)$ and trace estimates we obtain

$$
\frac{1}{2} \int_{\Gamma} \boldsymbol{V}_{1} \cdot \boldsymbol{n}|\hat{M}|^{2} d S \leq C_{1} \int_{\Omega}|\hat{M}|^{2} d \Omega+\varepsilon \int_{\Omega}|\nabla \hat{M}|^{2} d \Omega .
$$

For the second integral, using Hölder's inequality it follows that

$$
\begin{aligned}
\int_{\Omega} \hat{\boldsymbol{V}} \cdot \nabla \hat{M} M_{2} d \Omega & \leq\|\hat{\boldsymbol{V}}(t)\|_{\left[L^{2}(\Omega)\right]^{n}}\left\|M_{2}(t)\right\|_{L^{\infty}(\Omega \times(0, T))}\|\nabla \hat{M}(t)\| \\
& \leq C_{2}\|\hat{U}(t)\|^{2} \alpha^{2}(t)+\varepsilon\|\nabla \hat{M}(t)\|^{2},
\end{aligned}
$$

with $C_{2}>0$ independent of $T$. For the third integral we have

$$
\begin{aligned}
\int_{\Omega}\left[k_{1} M_{2}+k_{-1}\right] \hat{U} \hat{M} d \Omega & \leq\left(k_{1} \alpha(t)+k_{-1}\right)\|\hat{U}(t)\|\|\hat{M}(t)\| \\
& \leq \frac{1}{2}\left(k_{1} \alpha(t)+k_{-1}\right)^{2}\|\hat{U}(t)\|^{2}+\frac{1}{2}\|\hat{M}(t)\|^{2} .
\end{aligned}
$$

In conclusion, from the estimates (31)-(33) it follows that

$$
\begin{aligned}
\frac{1}{2} \frac{d}{d t}\|\hat{M}(t)\|^{2}+(D-2 \varepsilon)\|\nabla \hat{M}\|^{2} & \leq\left[C_{1}+\frac{1}{2}\right]\|\hat{M}(t)\|^{2} \\
& +\left[C_{2} \alpha^{2}(t)+\frac{1}{2}\left(k_{1} \alpha(t)+k_{-1}\right)^{2}\right]\|\hat{U}(t)\|^{2} .
\end{aligned}
$$


Choose $\varepsilon=D / 4$, integrate over $[0, t]$ and use (29) to obtain

$$
\|\hat{M}(t)\|^{2} \leq \gamma_{1}(t)\left\|\hat{M}^{\sharp}\right\|_{L^{2}\left(0, T ; L^{2}(\Omega)\right)}^{2}+C \int_{0}^{t}\|\hat{M}(s)\|^{2} d s,
$$

where $\gamma_{1}(t)=2 C_{2} \alpha^{2}(t)+\left(k_{1} \alpha(t)+k_{-1}\right)^{2}$ and $C=2 C_{1}+1$. Finally, using Gronwall's inequality we get

$$
\|\hat{M}(t)\|^{2} \leq e^{C t} \gamma_{1}(t)\left\|\hat{M}^{\sharp}\right\|_{L^{2}\left(0, T ; L^{2}(\Omega)\right)}^{2},
$$

and integrating on $[0, T]$ yields

$$
\begin{aligned}
\|\hat{M}\|_{L^{2}\left(0, T ; L^{2}(\Omega)\right)}^{2} & \leq \gamma(T)\left\|\hat{M}^{\sharp}\right\|_{L^{2}\left(0, T ; L^{2}(\Omega)\right)}^{2} \\
\gamma(T) & :=T e^{C T} \gamma_{1}(T)
\end{aligned}
$$

which implies the continuity of the operator $\mathcal{R}$.

4. $\mathcal{R}(\mathcal{K})$ is relatively compact in $L^{2}\left(0, T ; L^{2}(\Omega)\right)$. We will use Aubin's compactness theorem (see Theorem 5.1 in Lions [9], Section 5.5, pp. 57-64, and Tartar [14], Chapter 24, pp. 137-141). Suppose that the sequence $\left\{M_{n}^{\sharp}\right\}$ is uniformly bounded in $L^{2}\left(0, T ; L^{2}(\Omega)\right)$. Then by the continuity of $\mathcal{R}$ the sequence $\left\{\mathcal{R} M_{n}^{\sharp}=M_{n}\right\}$ is also uniformly bounded in $L^{2}\left(0, T ; L^{2}(\Omega)\right)$, and the estimates in Lemma 1 imply that $\left\{M_{n}\right\}$ is uniformly bounded in $L^{2}\left(0, T ; H^{1}(\Omega)\right)$. Furthermore, the sequence of derivatives $\left\{\partial_{t} M_{n}\right\}$ is uniformly bounded in $L^{2}\left(0, T ; H^{-1}(\Omega)\right)$. Indeed, we have

$$
\begin{aligned}
\int_{0}^{T} \int_{\Omega}\left|\boldsymbol{V}_{n} M_{n}\right|^{2} d \Omega d t & \leq T\left\|M_{n}\right\|_{L^{\infty}(\Omega \times(0, T))}\left\|\boldsymbol{V}_{n}\right\|_{L^{\infty}\left(0, T ;\left[L^{2}(\Omega)\right]^{n}\right)}^{2} \\
& \leq C T \alpha^{2}(T)\|a\|_{\infty}\|A\|_{\infty},
\end{aligned}
$$

from where it follows that for all $t \in[0, T]$ the expression

$$
\partial_{t} M_{n}=\nabla \cdot\left[D \nabla M_{n}-\boldsymbol{V}_{n} M_{n}\right]-k_{1} M_{n} U_{n}+k_{-1}\left[A-U_{n}\right]
$$

defines a uniformly bounded sequence of distributions in $H^{1}(\Omega)$. Therefore, applying Aubin's theorem to the spaces $H^{1}(\Omega) \subset L^{2}(\Omega) \subset H^{-1}(\Omega)$ we obtain that the sequence $\left\{M_{n}\right\}$ is relatively compact in $L^{2}\left(0, T ; L^{2}(\Omega)\right)$.

\subsection{Conclusion of the proof}

Lemma 3 For any $T>0$ the reaction-diffusion system (18)-(20) has global unique weak solutions $M(\boldsymbol{x}, t), U(\boldsymbol{x}, t)$ and $\boldsymbol{V}(\boldsymbol{x}, t)$ in $\Omega \times(0, T)$ a.e. Furthermore, $M(\boldsymbol{x}, t) \geq 0$ a.e. in $\Omega \times(0, T)$, and the estimates of Lemma 1 hold.

Proof: The four statements of Lemma 2 imply that we can apply Schauder's Fixed Point Theorem to the operator $\mathcal{R}$ and obtain a fixed point $M^{\sharp}(\boldsymbol{x}, t)=M(\boldsymbol{x}, t)$ in $\mathcal{K}$. This implies that $M(\boldsymbol{x}, t) \geq 0$ a.e. in $\Omega \times(0, T)$, and in consequence Lemma 1 holds.

Observing carefully the explicit expression of $\gamma(T)$ in (34) it follows that $\gamma(T) \rightarrow 0$ if $T \rightarrow 0$. Therefore, the operator $\mathcal{R}$ is a contraction if $T>0$ is small enough, and in consequence we have the local uniqueness of (18)-(20). 
Now choose a time $T_{0} \in(0, T)$ such that $\gamma\left(T_{0}\right)<1$ and perform the very same calculations we have already made but with initial conditions $M\left(\boldsymbol{x}, T_{0}\right)$ and $U\left(\boldsymbol{x}, T_{0}\right)$ instead of $m_{0}(\boldsymbol{x})$ and $A(\boldsymbol{x})$, respectively. This yields a different set of bounds

$$
\begin{aligned}
\alpha\left(T_{0}, t\right) & :=\left\|M\left(T_{0}\right)\right\|_{\infty}+k_{1}\left(t-T_{0}\right)\left\|U\left(T_{0}\right)\right\|_{\infty}, \\
\beta\left(T_{0}, t\right) & :=k_{1}^{2}\left\|U\left(T_{0}\right)\right\|_{\infty}^{2}\left(t-T_{0}\right), \\
\gamma_{1}\left(T_{0}, t\right) & :=2 C_{2} \alpha^{2}\left(T_{0}, t\right)+\left(k_{1} \alpha\left(T_{0}, t\right)+k_{-1}\right)^{2}, \\
\gamma\left(T_{0}, t\right) & :=\left(t-T_{0}\right) e^{C\left(t-T_{0}\right)} \gamma_{1}\left(T_{0}, t\right) .
\end{aligned}
$$

Recall that $U\left(T_{0}\right) \leq A(\boldsymbol{x})$ and $\|M\|_{\infty} \leq \alpha(T)$ and define

$$
\begin{aligned}
\widetilde{\alpha}(T) & :=\alpha(T)+k_{1} t\|A\|_{\infty}, \\
\widetilde{\beta}(T) & :=\beta(T), \\
\widetilde{\gamma}_{1}(T) & :=2 C_{2} \widetilde{\alpha}^{2}(T)+\left(k_{1} \widetilde{\alpha}(T)+k_{-1}\right)^{2}, \\
\widetilde{\gamma}(T) & :=T e^{C T} \widetilde{\gamma}_{1}(T) .
\end{aligned}
$$

These new bounds are independent of the initial conditions $M\left(T_{0}\right)$ and $U\left(T_{0}\right)$. Therefore, if $T_{0}$ was chosen such that $\widetilde{\gamma}\left(T_{0}\right)<1$ we can extend the uniqueness result to the interval $\left[T_{0}, 2 T_{0}\right]$, and repeating this procedure we obtain uniqueness on the whole interval $[0, T]$, i.e. global uniqueness.

From Lemmas 1 and 3 the proof of Theorem 1 follows immediately.

\section{Proof of Theorem 2}

\subsection{A priori estimates}

Lemma 4 If $U(\boldsymbol{x}, t)$ and $W(\boldsymbol{x}, t)$ are solutions of (21)-(23) then $U, W \in L^{\infty}(\Omega \times(0, T))$ and

$$
0 \leq U(\boldsymbol{x}, t)+W(\boldsymbol{x}, t) \leq\|A\|_{\infty} \quad \text { a.e. }
$$

Proof: $\quad$ For any $c \in \mathbb{R}$ the function $Y:=U+W-c$ satisfies

$$
\partial_{t} Y=d \triangle Y, \quad \frac{\partial Y}{\partial \boldsymbol{n}}=0 \quad \text { on } \Gamma, \quad Y(\boldsymbol{x}, 0)=A(\boldsymbol{x})-c .
$$

Therefore, applying the Maximum Principle to the cases $c=0$ and $c=\|A\|_{\infty}$ we obtain the first and second inequalities in (35), respectively.

Lemma 5 If $M(\boldsymbol{x}, t)$ is a solution of (21)-(23) such that $M(\boldsymbol{x}, t) \geq 0$ a.e. in $\Omega \times(0, T)$ then:

1. $M \in L^{\infty}(\Omega \times(0, T))$ and $0 \leq M(\boldsymbol{x}, t) \leq \alpha(t):=\left\|m_{0}\right\|_{\infty}+k_{-1} t\|A\|_{\infty}$ a.e. in $\Omega \times(0, T)$.

2. $M \in L^{\infty}\left(0, T ; H^{1}(\Omega)\right)$ and

$$
\|M(t)\|^{2}+D \int_{0}^{t} e^{C(t-s)}\|\nabla M(s)\|^{2} d s \leq e^{C t}\left[\left\|m_{0}\right\|^{2}+k_{-1}^{2} t\|A\|^{2}\right] .
$$


3. $U, W \in L^{\infty}(\Omega \times(0, T))$ and $0 \leq U(\boldsymbol{x}, t)+W(\boldsymbol{x}, t) \leq A(\boldsymbol{x})$ a.e. in $\Omega \times(0, T)$.

4. $U, W \in L^{\infty}\left(0, T ; H^{1}(\Omega)\right)$ and

$$
\|U(t)\|^{2}+\|W(t)\|^{2}+2 d \int_{0}^{t} e^{\int_{s}^{t} c(r) d r}\left(\|\nabla U(s)\|^{2}+\|\nabla W(s)\|^{2}\right) d s \leq e^{\int_{0}^{t} c(s) d s}\|A\|^{2},
$$

where $c(t)=2\left[k_{-1}+k_{1} \alpha(t)\right]$.

5. $\boldsymbol{V} \in L^{\infty}\left(0, T ;\left[L^{2}(\Omega)\right]^{n}\right)$ and $\|\boldsymbol{V}\|_{L^{\infty}\left(0, T ;\left[L^{2}(\Omega)\right]^{n}\right)} \leq C\|a\|_{\infty}\|A\|_{\infty}$.

Proof: The only statements we need to prove are 3 and 4 because the other ones can be proved using exactly the same arguments we have already performed in Section 3.

Let us first prove statement 3. An integration by parts in (21)-(23) yields

$$
\frac{1}{2} \frac{d}{d t}\left\|U^{-}\right\|^{2}+d\left\|\nabla U^{-}\right\|^{2}+k_{1} \int_{\Omega} M\left|U^{-}\right|^{2}=-k_{-1} \int_{\Omega} W U^{-} d \Omega .
$$

We affirm that $W U^{-} \geq 0$. Indeed, by Lemma 4 we have that $U+W \geq 0$, which implies that $0 \leq$ $U^{-}(U+W)=-\left|U^{-}\right|^{2}+U^{-} W$. Therefore $U^{-} W \geq 0$, and in consequence $\left\|U^{-}(t)\right\|^{2} \equiv 0$. The argument for proving $\left\|W^{-}(t)\right\|^{2} \equiv 0$ is the same.

For statement 4 , integration by parts yields

$$
\begin{aligned}
\frac{1}{2} \frac{d}{d t}\|U\|^{2}+d\|\nabla U\|^{2}+k_{1} \int_{\Omega} M|U|^{2} d \Omega & =k_{-1} \int_{\Omega} W U d \Omega, \\
\frac{1}{2} \frac{d}{d t}\|W\|^{2}+d\|\nabla W\|^{2}+k_{-1} \int_{\Omega}|W|^{2} d \Omega & =k_{1} \int_{\Omega} M U W d \Omega .
\end{aligned}
$$

Adding both equalities we get

$$
\frac{1}{2} \frac{d}{d t}\left[\|U\|^{2}+\|W\|^{2}\right]+d\left[\|\nabla U\|^{2}+\|\nabla W\|^{2}\right] \leq \frac{c(t)}{2}\|U\|\|W\|,
$$

where $c(t)=2\left[k_{-1}+k_{1} \alpha(t)\right]$. Multiplying this inequality by $e^{-\int_{0}^{t} c(s) d s}$ and integrating on $[0, T]$ we obtain the result.

\subsection{The Fixed Point operator}

Define

$$
\mathcal{K}:=\left\{M \in L^{2}\left(0, T ; L^{2}(\Omega)\right): 0 \leq M(\boldsymbol{x}, t) \leq\left\|m_{0}\right\|_{\infty}+k_{-1} t\|A\|_{\infty} \quad \text { a.e. in } \Omega \times(0, T)\right\} .
$$

Fix $M^{\sharp} \in \mathcal{K}$ and set

$$
\begin{aligned}
\partial_{t} U=d \triangle U-k_{1} M^{\sharp} U+k_{-1} W & \text { in } \Omega \times(0, T), \\
\partial_{t} W=d \triangle U+k_{1} M^{\sharp} U-k_{-1} W & \text { in } \Omega \times(0, T), \\
\nabla U \cdot \mathbf{n}(\sigma, t)=0 & \text { on } \Gamma \times[0, T], \\
\nabla W \cdot \mathbf{n}(\sigma, t)=0 & \text { on } \Gamma \times[0, T] .
\end{aligned}
$$


For any finite time interval $[0, T]$ this linear system has unique solutions $U(\boldsymbol{x}, t)$ and $W(\boldsymbol{x}, t)$, which are non-negative and satisfy $0 \leq U(\boldsymbol{x}, t)+W(\boldsymbol{x}, t) \leq A(\boldsymbol{x})$ a.e. in $\Omega \times(0, T)$. With these $U(\boldsymbol{x}, t)$ and $W(\boldsymbol{x}, t)$ set

$$
\left\{\begin{aligned}
\triangle \phi(\boldsymbol{x}, t) & =0 & & \text { in } \Omega \\
\nabla \phi \cdot \boldsymbol{n}(\sigma, t) & =a(\sigma) \int_{\Omega} W(\boldsymbol{x}, t) d \Omega & & \text { on } \Gamma
\end{aligned}\right.
$$

with $a(\sigma) \in L^{\infty}(\Gamma)$ and $\int_{\Omega} \phi(\boldsymbol{x}, t) d \Omega=\int_{\Gamma} a(\sigma) d S=0$. This linear problem has a unique solution $\phi(\boldsymbol{x}, t) \in L^{\infty}\left(0, T ; H^{1}(\Omega)\right)$ satisfying

$$
\|\phi\|_{H^{1}(\Omega)} \leq C\|a\|_{\infty}\|A\|_{\infty}
$$

Now, define $\boldsymbol{V}(\boldsymbol{x}, t)=\nabla \phi(\boldsymbol{x}, t)$ and set

$$
\begin{aligned}
\partial_{t} M=\nabla \cdot[D \nabla M-\boldsymbol{V} M]-k_{1} M U+k_{-1}[A-U] & \text { in } \Omega \times(0, T), \\
M(\sigma, t)=0 & \text { on } \Gamma_{a} \times[0, T], \\
\nabla M \cdot \mathbf{n}(\sigma, t)=0 & \text { on } \Gamma_{r} \times[0, T], \\
M(\boldsymbol{x}, 0)=m_{0}(\boldsymbol{x}) & \text { in } \Omega .
\end{aligned}
$$

For any finite time interval $[0, T]$ there exists a unique weak solution $M(\boldsymbol{x}, t)$ such that $0 \leq M(\boldsymbol{x}, t) \leq$ $\left\|m_{0}\right\|_{\infty}+k_{-1} t\|A\|_{\infty}$ a.e. in $\Omega \times(0, T)$.

We have just constructed a chain of maps $M^{\sharp} \mapsto(U, W) \mapsto \boldsymbol{V} \mapsto M$, and our goal is to show that the operator $\mathcal{R}\left(M^{\sharp}\right):=M$ has a fixed point.

\subsection{Conclusion of the proof}

Let $M_{1}^{\sharp}, M_{2}^{\sharp} \in \mathcal{K}$, and for each $i=1,2$ consider the chain of maps

$$
M_{i}^{\sharp} \longmapsto U_{i} \longmapsto \boldsymbol{V}_{i} \longmapsto M_{i}
$$

Define $\hat{M}^{\sharp}:=M_{2}^{\sharp}-M_{1}^{\sharp}, \hat{U}:=U_{2}-U_{1}, \hat{W}:=W_{2}-W_{1}, \hat{\varphi}:=\varphi_{2}-\varphi_{1}, \hat{\boldsymbol{V}}:=\boldsymbol{V}_{2}-\boldsymbol{V}_{1}$ and $\hat{M}:=M_{2}-M_{1}$. The differences $\hat{M}, \hat{U}$ and $\hat{W}$ solve the equations

$$
\begin{aligned}
\partial_{t} \hat{M} & =\nabla \cdot\left[D \nabla \hat{M}-\boldsymbol{V}_{1} \hat{M}-\hat{\boldsymbol{V}} M_{2}\right]-k_{1} \hat{U} M_{2}-k_{1} U_{1} \hat{M}+k_{-1} \hat{W}, \\
\partial_{t} \hat{U} & =d \triangle \hat{U}-k_{1} \hat{U} M_{2}^{\sharp}-k_{1} U_{1} \hat{M}^{\sharp}+k_{-1} \hat{W}, \\
\partial_{t} \hat{W} & =d \triangle \hat{U} k_{1} \hat{U} M_{2}^{\sharp}+k_{1} U_{1} \hat{M}^{\sharp}-k_{-1} \hat{W},
\end{aligned}
$$

with homogeneous initial and boundary conditions, whereas $\hat{\boldsymbol{V}}=\nabla \hat{\phi}$ solves

$$
\begin{aligned}
\nabla \cdot \hat{\boldsymbol{V}} & =0 \\
\hat{\boldsymbol{V}} \cdot \boldsymbol{n} & =a \int_{\Omega} \hat{W} d \Omega .
\end{aligned}
$$


Lemma 6 If for $i=1,2, M_{i}^{\sharp}(\boldsymbol{x}, t) \geq 0$ a.e. in $\Omega \times(0, T)$ then there exist positive continuous functions $C_{1}(t), C_{2}(t)$ and $C_{3}(t)$ such that

$$
\begin{aligned}
\frac{d}{d t}\|\hat{M}(t)\|^{2} & \leq C_{1}(t)\left(\|\hat{M}(t)\|^{2}+\|\hat{U}(t)\|^{2}+\|\hat{W}(t)\|^{2}\right) . \\
\frac{d}{d t}\left(\|\hat{U}(t)\|^{2}+\|\hat{W}(t)\|^{2}\right) & \leq C_{2}(t)\left(\|\hat{U}(t)\|^{2}+\|\hat{W}(t)\|^{2}+\left\|\hat{M}^{\sharp}(t)\right\|^{2}\right), \\
\frac{d}{d t}\left(\|\hat{U}(t)\|^{2}+\|\hat{W}(t)\|^{2}\right) & \leq C_{3}(t)\left(\|\hat{U}(t)\|^{2}+\|\hat{W}(t)\|^{2}\right) .
\end{aligned}
$$

Proof: Multiply (37) by $\hat{M}$ and integrate by parts to get

$$
\begin{aligned}
\frac{1}{2} \frac{d}{d t}\|\hat{M}\|^{2}+D\|\nabla \hat{M}\|^{2} & =-k_{1} \int_{\Omega}|\hat{U}|^{2} \hat{\boldsymbol{V}}_{1} \cdot \boldsymbol{n} d S-\int_{\Omega} M_{2} \hat{\boldsymbol{V}} \cdot \nabla \hat{M} d \Omega \\
& -k_{1} \int_{\Omega} \hat{U} M_{2} \hat{M} d \Omega-k_{1} \int_{\Omega} U_{1}|\hat{M}|^{2} d \Omega+k_{-1} \int_{\Omega} \hat{W} \hat{M}
\end{aligned}
$$

Noticing that all the functions are in $L^{\infty}$ we can deduce the first estimate in (38). Indeed, since $\|\hat{\boldsymbol{V}}\| \leq$ $C\|\hat{W}\|$ it follows that

$$
\int_{\Omega} M_{2} \hat{\boldsymbol{V}} \cdot \nabla \hat{M} d \Omega \leq \varepsilon\|\nabla \hat{M}\|^{2}+C(t)\|\hat{W}\|^{2},
$$

and the other four integrals in (39) can be estimated similarly in order to get

$$
\frac{d}{d t}\|\hat{M}(t)\|^{2} \leq C_{1}(t)\left(\|\hat{M}(t)\|^{2}+\|\hat{U}(t)\|^{2}+\|\hat{W}(t)\|^{2}\right) .
$$

Multiply (37) by $\hat{U}$ and integrate by parts to get

$$
\frac{1}{2} \frac{d}{d t}\|\hat{U}\|^{2}+d\|\nabla \hat{U}\|^{2}=-k_{1} \int_{\Omega}|\hat{U}|^{2} M_{2}^{\sharp} d \Omega-k_{1} \int_{\Omega} U_{1} \hat{M}^{\sharp} \hat{U} d \Omega+k_{1} \int_{\Omega} \hat{W} \hat{U} d \Omega .
$$

Observe that second integral in (40) can be estimated in two ways, either

$$
\int_{\Omega} U_{1} \hat{M}^{\sharp} \hat{U} d \Omega \leq C\left(\|\hat{U}(t)\|^{2}+\left\|\hat{M}^{\sharp}(t)\right\|^{2}\right)
$$

or either

$$
\int_{\Omega} U_{1} \hat{M}^{\sharp} \hat{U} d \Omega \leq C(t)\|\hat{U}(t)\|^{2} .
$$

In the first case we can deduce that

$$
\frac{d}{d t}\|\hat{U}(t)\|^{2} \leq C(t)\left(\|\hat{U}(t)\|^{2}+\|\hat{W}(t)\|^{2}+\left\|\hat{M}^{\sharp}(t)\right\|^{2}\right),
$$

whereas in the second case we can show that

$$
\frac{d}{d t}\|\hat{M}(t)\|^{2} \leq C(t)\left(\|\hat{U}(t)\|^{2}+\|\hat{W}(t)\|^{2}\right) .
$$

Now perform the same estimates for $\hat{W}$ and add up both the estimates for $\hat{U}$ and $\hat{W}$. It follows then that with estimates of type (41) we obtain the second inequality in (38), whereas with estimates of type (42) we get the third inequality in (38). 
Lemma 7 For any $T>0$ the reaction-diffusion system (21)-(23) is a well-posed problem, i.e. it has global unique weak solutions $M(\boldsymbol{x}, t), U(\boldsymbol{x}, t), W(\boldsymbol{x}, t)$ and $\boldsymbol{V}(\boldsymbol{x}, t)$ which depend continuously on the initial data. Moreover, $M(\boldsymbol{x}, t), U(\boldsymbol{x}, t)$ and $W(\boldsymbol{x}, t)$ are non-negative a.e. in $\Omega \times(0, T)$ and the estimates of Lemma 5 hold.

Proof: Using Gronwall's lemma in the second equation in (38) we have

$$
\|\hat{U}(t)\|^{2}+\|\hat{W}(t)\|^{2} \leq C_{2}(t)\left\|\hat{M}^{\sharp}(t)\right\|_{L^{2}\left(0, T ; L^{2}(\Omega)\right)}^{2} .
$$

Plugging this inequality into the first equation in (38) and using again Gronwall's lemma we obtain that there exists a positive continuous function $\theta(t)$ such that

$$
\|\hat{M}(t)\|^{2} \leq \theta(t)\left\|\hat{M}^{\sharp}(t)\right\|_{L^{2}\left(0, T ; L^{2}(\Omega)\right)}^{2} .
$$

Integrating on $[0, T]$ we have

$$
\|\hat{M}(t)\|_{L^{2}\left(0, T ; L^{2}(\Omega)\right)}^{2} \leq T \theta(T)\left\|\hat{M}^{\sharp}(t)\right\|_{L^{2}\left(0, T ; L^{2}(\Omega)\right)}^{2},
$$

which implies that the operator $\mathcal{R}$ is continuous. Therefore, applying Schauder's Fixed Point Theorem it follows that $\mathcal{R}$ has a fixed point $M(\boldsymbol{x}, t)$. With this $M(\boldsymbol{x}, t)$ we can construct $U(\boldsymbol{x}, t), W(\boldsymbol{x}, t)$ and $\boldsymbol{V}(\boldsymbol{x}, t)$, and the four of them are global solutions of the problem (21)-(23). Moreover, since $M(\boldsymbol{x}, t) \geq 0$ a.e. in $\Omega \times(0, T)$ then the estimates of Lemma 5 hold.

Now suppose we have non-homogeneous initial conditions. Then adding up the first and third equations in (38) and using Gronwall's lemma we can show that there exists a positive continuous function $\kappa(t)$ such that

$$
\|\hat{M}(t)\|^{2}+\|\hat{U}(t)\|^{2}+\|\hat{W}(t)\|^{2} \leq \kappa(t)\left(\|\hat{M}(0)\|^{2}+\|\hat{U}(0)\|^{2}+\|\hat{W}(0)\|^{2}\right) .
$$

Therefore, the solutions are unique and depend continuously on the initial data.

From Lemmas 5 and 7 the proof of Theorem 2 follows immediately.

\section{$5 \quad$ Proof of Theorem 3}

For any $0<d \leq d_{0}$ let $\left(M^{d}, U^{d}, W^{d}, \mathbf{V}^{d}\right)$ be the weak solutions of (21)-(23) and let $d \rightarrow 0$. First, Lemma 5 implies that the sequence $U^{d}$ is bounded in

$$
\mathcal{X}:=L^{2}\left(0, T ; L^{2}(\Omega)\right) \cap L^{\infty}(\Omega \times(0, T)),
$$

which implies that a subsequence, still denoted $U^{d}$, converges weakly- $\star$ in $\mathcal{X}$ to a limit $U^{0}$. Similarly, a subsequence $W^{d}$ converges weakly- $\star$ in $\mathcal{X}$ to a limit $W^{0}$.

Second, Lemma 5 also affirms that the sequence $\mathbf{V}^{d}$ is uniformly bounded in

$$
\mathcal{Y}:=L^{\infty}\left(0, T ;\left[L^{2}(\Omega)\right]^{n}\right)
$$

then there is a subsequence $\mathbf{V}^{d}$ converging weakly- $\star$ in $\mathcal{Y}$ to a limit $\mathbf{V}^{0}$. 
Third, from Lemma 5 the sequence $M^{d}$ is uniformly bounded in

$$
\mathcal{Z}:=L^{2}\left(0, T ; L^{2}(\Omega)\right) \cap L^{2}\left(0, T ; H^{1}(\Omega)\right) \cap L^{\infty}\left(0, T ; H^{1}(\Omega)\right) \cap L^{\infty}(\Omega \times(0, T)),
$$

hence a subsequence $M^{d}$ converges weakly-^ in $\mathcal{Z}$ to a limit $M^{0}$. Moreover, using classical estimates of type

$$
\int_{0}^{T} \int_{\Omega}\left|\mathbf{V}^{d} M^{d}-\mathbf{V}^{0} M^{0}\right| d \Omega d t \leq C_{1}\left\|\mathbf{V}^{d}-\mathbf{V}^{0}\right\|+C_{2}\left\|M^{d}-M^{0}\right\|
$$

it follows that $\mathbf{V}^{d} M^{d} \rightarrow \mathbf{V}^{0} M^{0}$ strongly in $\left[L^{1}(\Omega \times(0, T))\right]^{n}$ and $M^{d} U^{d} \rightarrow M^{0} U^{0}$ strongly in $L^{1}(\Omega \times$ $(0, T))$. This implies that the sequence $\partial_{t} M^{d}$ is uniformly bounded in $L^{2}\left(0, T ; H^{-1}(\Omega)\right)$, so applying Aubin's compactness theorem we have that the convergence $M^{d} \rightarrow M^{0}$ is in fact strong in $L^{2}\left(0, T ; L^{2}(\Omega)\right)$.

In the light of the former convergences we obtain that a subsequence $\left(M^{d}, U^{d}, W^{d}, \mathbf{V}^{d}\right)$ of weak solutions of (21)-(23) converges weakly in $L^{2}\left(0, T ; L^{2}(\Omega)\right)$ to $\left(M^{0}, U^{0}, W^{0}, \mathbf{V}^{0}\right)$, which is a weak solution of (18)-(20). However, the uniqueness of the problem (18)-(20) implies, on the one hand, that $\left(M^{0}, U^{0}, W^{0}, \mathbf{V}^{0}\right)=(M, U, W, \mathbf{V})$, and on the other hand, that the whole original sequence $\left(M^{d}, U^{d}, W^{d}, \mathbf{V}^{d}\right)$ converges weakly in $L^{2}\left(0, T ; L^{2}(\Omega)\right)$. Moreover, since in the limit we have $\partial_{t}(U+W)=0$ and $U, W \in$ $L^{\infty}(\Omega \times(0, T))$ then $U(\boldsymbol{x}, t)+W(\boldsymbol{x}, t)=A(\boldsymbol{x})$ a.e. in $\Omega \times(0, T)$.

This concludes the proof of Theorem 3 .

\section{Final remarks}

\subsection{On the cytoplasmic flux}

Throughout this article we have supposed that the cytoplasmic flow field $\boldsymbol{V}$ is incompressible $(\nabla \cdot \boldsymbol{V}=0)$ and that it comes from a potential $(\boldsymbol{V}=\nabla \phi)$. These two hypotheses are assumed in the model of Holcman and Schuss [7], but the cytoplasmic flow could have been modeled in a more realistic way without affecting the results we presented. Indeed, we could consider that $\boldsymbol{V}$ follows Stokes' equation

$$
\begin{aligned}
-\mu \Delta \boldsymbol{V}+\nabla p=0 & \text { in } \Omega, \\
\nabla \cdot \boldsymbol{V}=0 & \text { in } \Omega, \\
\boldsymbol{V}=\lambda(t) \boldsymbol{f} & \text { on } \Gamma,
\end{aligned}
$$

with $\boldsymbol{f} \in\left[H^{1 / 2}(\Gamma) \cap L^{\infty}(\Gamma)\right]^{n}$. Under these assumptions the problem (43) has a unique solution $\boldsymbol{V} \in$ $\left[H^{1}(\Omega)\right]^{n}$ satisfying

$$
\|\boldsymbol{V}\|_{\left[H^{1}(\Omega)\right]^{n}} \leq C|\lambda(t)| \cdot\|\boldsymbol{f}\|_{\left[H^{1 / 2}(\Gamma)\right]^{n}}
$$

which can be used instead of (17) to obtain the same results of Theorems 1, 2 and 3.

\subsection{On the diffusion coefficient}

If we wish to take into account the existence of obstacles inside the spine, like organelles or macromolecules, we can add them in two forms: either as "exterior domains", i.e. we take out a tiny section from the domain $\Omega$ and suppose that the boundary of the section belongs to the boundary of $\Omega$, or either 
by considering that the diffusion coefficients are no longer constant. The results we presented here are still valid in both situations provided that the exterior domains have $C^{1}$ boundaries, $D, d \in C^{1}(\bar{\Omega} \times[0, T])$ and

$$
0<D_{1} \leq D(\boldsymbol{x}, t) \leq D_{2}, \quad 0<d_{1} \leq d(\boldsymbol{x}, t) \leq d_{2} .
$$

\subsection{On the reactions between calcium and the proteins}

As in Holcman and Schuss [7], we assumed in the model that all binding sites have the same affinity for the $\mathrm{Ca}^{2+}$ ions, but this is not the real case. Indeed, calcineurin has one binding site with high affinity and three with low affinity (see Klee et al [8]), AM-type proteins like Troponin have two low affinity sites and two high affinity sites (see Farah et al [3]), and $\mathrm{CaM}$ with two $\mathrm{Ca}^{2+}$ ions bound has more affinity to bind calcium than CaM with no $\mathrm{Ca}^{2+}$ ions bound (see Shiftman et al [13]). Nevertheless, such distinctions were not considered here in order to keep things as simple as possible.

\section{Discussion}

All the results we presented here are new and can be considered as the sequel of the works of Holcman et al [6] and [7], and in particular of [7], where Holcman and Schuss proposed the reaction-diffusion system (5) as a model for calcium dynamics inside a dendritic spine. Our main results are Theorem 1, where we proved that the system (5) in its modified form (18)-(20) has global unique positive solutions, Theorem 2 , where we proved that if the proteins diffuse then the corresponding problem (21)-(23) is well-posed, and Theorem 3, where we showed that the solutions of (21)-(23) converge to the solutions of (18)-(20) when $d \rightarrow 0$.

We mentioned also that the experimental evidence suggests that the twitching motion of the spine should depend on the total number of occupied binding sites. We made the assumption that the spine twitching depends on the cytoplasmic velocity $\boldsymbol{V}$ at the spine surface $\Gamma$, and that this value depends on the total number of $\mathrm{Ca}^{2+}$ ions that are bound to the proteins. This renders a strong coupling between $M, U, W$ and $\boldsymbol{V}$ but nevertheless we succeeded to solve this coupled system.

The hypothesis of diffusion in the proteins makes the problem easier because for $d>0$ the problem (21)-(23) is strongly elliptic, whereas for $d=0$ the problem (18)-(20) is still elliptic but degenerate. This fact explains, or at least justifies, why in the case of fixed proteins we were not able to prove the continuous dependence of the solutions on the initial data.

There are at least two tasks that we consider interesting to address in the future. First, it could be illustrative to perform numerical simulations for both reaction-diffusion models (18)-(20) and (21)-(23) in order to compare them with the simulations of the Langevin equation (1) that appeared in Holcman et al [6], [7], and also with experimental data. Second, the questions of stability and behavior of the solutions at large times are completely open.

\section{References}

[1] Alberts B. et al. (2002) Molecular Biology of the cell, 4th ed. Garland Science, USA.

[2] Evans L. (1998) Partial differential equations. American Mathematical Society, USA.

[3] Farah C.S., Reinach F.C. (1995) FASEB Journal Vol.9, pp. 755-767. 
[4] Sneyd, J. (editor) (2005) Tutorial in Mathematical Biosciences Vol.II, Mathematical modeling of calcium dynamics and signal transduction, Lecture Notes Math, No. 1867.

[5] Haines, D. (2002) Fundamental Neuroscience, 2nd ed. Churchill Livingstone, USA.

[6] Holcman D., Schuss Z., Korkotian E. (2004) Calcium dynamics in dendritic spines and spine motility. Biophysical Journal Vol. 87, p. 81-91.

[7] Holcman D., Schuss Z. (2005) Modeling calcium dynamics in dendritic spines. SIAM J. Appl. Math. Vol. 65 No. 3, pp. 1006-1026.

[8] Klee C.B., Ren H., Wang X. (1998) J. Biol. Chem. Vol. 273, Issue 22, pp. 13367-13370.

[9] Lions J.L. (1969) Quelques méthodes de résolution des problèmes aux limites nonlinéaires. Dunod, Paris, France.

[10] Nimchinsky E.A., Sabatini B.L., Svoboda K. (2002) Structure and function of dendritic spines. Annu. Rev. Phisiol. Vol. 64, pp. 313-353.

[11] Øksendal B. (2003) Stochastic differential equations, 6th ed. Springer-Verlag, USA.

[12] Purves D. et al (2004) Neuroscience, 3rd. ed., Sinauer, USA.

[13] Shiftman, J.M., Choi M.H., Mihalas S., Mayo S.L., Kennedy M.B. (2006) PNAS Vol. 103, No. 38, pp. 13968-13973.

[14] Tartar L. (2006) An introduction to Navier-Stokes equation and Oceanography. Springer-Verlag, Netherlands.

[15] Taylor M. (1997) Partial differential equations III. Nonlinear equations. Springer-Verlag, USA. 